\title{
REDACCIÓN Y TRAMITACIÓN PARLAMENTARIA DE LA PRIMERA LEY ELECTORAL EN USA (1789)
}

\section{DRAFTING AND PARLIAMENTARY APPROVAL OF THE FIRST APPORTIONMENT BILL OF THE US (1789)}

\author{
María Corrés Illera \\ Universidad Complutense de Madrid
}

\begin{abstract}
SUMARIO: I: INTRODUCCIÓN- II. TRAMITACIÓN EN LA PRIMERA CÁMARA DEL CONGRESO (CÁMARA DE REPRESENTANTES) - 1. Propuesta de ley 2. Reformas en el Comité de la Cámara de Representantes - 3. Reformas en el Comité de la Cámara de Representantes - 4. Votación en el pleno de la Cámara de Representantes - III. TRAMITACIÓN EN LA SEGUNDA CÁMARA DEL CONGRESO (SENADO).- IV. COMISIÓN MIXTA V. APROBACIÓN DEL TEXTO FINAL - VI. RATIFICACIÓN
\end{abstract}

Resumen: El presente trabajo analiza los cambios de redacción que experimentó la primera propuesta de ley electoral en EEUU, así como la evolución parlamentaria por la que atravesó hasta obtener la aprobación del Congreso. La propuesta inicial de Madison, fue enmendada a su paso por las distintas fases de la tramitación parlamentaria. La redacción final fue incluida en 1789 como enmienda I a la Constitución de EEUU, en lo que se conoce como Carta de Derechos Fundamentales

Abstract: This paper analyses the changes in the drafting on the first US apportionment bill. As well as the parliamentary stages it had to surmount in order to obtain Congressional approval. Madison's proposal was amended as it passed through the different stages of parliamentary proceedings. The finalized text was included in 1789 as Amendment I to the US Const. As such was presented for ratification within the Bill of Rights.

Palabras clave: Bill of Rights, Representación proporcional, enmienda, Constitución, Congreso

Keywords: Representatives, proportional, amendment, Constitution, Congress

\section{INTRODUCCIÓN}

Una de las condiciones que permitió la ratificación de la Constitución, fue la promesa inminente de una Carta de Derechos Fundamentales, si la Constitución se convertía en realidad.

Razón por la cual fue uno de los primeros asuntos abordados por el primer Congreso Federal, del cual nació una propuesta respaldada por el entonces miembro de la Cámara de Representantes, James Madison, con doce enmiendas a la Constitución para ser ratificadas por los Estados. De estas doce enmiendas sólo las últimas diez fueron ratificadas, convirtiéndose así en las diez primeras enmiendas de la Constitución y en lo que se ha venido a denominar la Carta de Derechos Fundamentales (Bill of Rights). 
Lo interesante es que en el proyecto inicial de enmienda, había un preámbulo y dos artículos que afectaban al Congreso y que no fueron ratificados por los estados en el siglo XVIII.

Esta última puntualización es necesaria porque una de las enmiendas presentadas en 1789 para la ratificación de los estados, y que sin embargo no obtuvo entonces el apoyo suficiente para ser ratificada, hoy sí forma parte de la Constitución de los Estados Unidos como enmienda XXVII, al llegar a ser ratificada en 1992 .

Entre los artículos no ratificados había uno que reformaba la forma de distribuir los asientos en el Congreso, por lo que podría considerarse el primer intento de reforma del sistema de reparto de escaños de la Cámara de Representantes, convirtiéndolo así en el primer proyecto de Ley Electoral aprobado por el Congreso de los Estados Unidos de América para el prorrateo de la Cámara.

\section{TRAMITACIÓN LEGISLATIVA EN LA PRIMERA CÁMARA DEL CONGRESO (CÁMARA DE REPRESENTANTES)}

\section{Propuesta de ley}

E1 8 de junio de 1789, durante el Primer Congreso Federal, James Madison ${ }^{1}$ propuso enmendar la Constitución recién entrada en vigor, con lo que denominó "Carta de Derechos Fundamentales" (Bill of Rights).

El documento inicial fue presentado al pleno de la Cámara de Representantes con un contenido de nueve enmiendas a la Constitución. ${ }^{2}$

E1 21 de julio de 1789, se asignó el asunto a un comité, compuesto por once miembros, uno por cada estado, teniendo en cuenta que North Carolina y Rhode Island aún no habian ratificado la Constitución. Entre los miembros de este comité estaban: James Madison como representante del estado de Virginia, Roger Sherman como el representante de Connecticut, y Elbridge Gerry como representante por Massachusetts. ${ }^{3}$

La segunda enmienda de Madison iba dirigida a reformar el sistema de reparto de escaños en la Cámara de Representantes teniendo en cuenta la elaboración del futuro censo en 1790. Madison consideraba que no tenía sentido mantener un precepto que aludiera al futuro primer censo, cuando éste ya se habria realizado.

Por lo que la reforma iba dirigida a subsanar los resultados del futurible censo, con la producción de los resultados del primer censo, más la interpretación de los mismos. Quedando el texto de la siguiente forma:

"Secondly. That in article 1st, section 2, clause 3, these words be struck out, to wit: 'The number of Representatives shall not exceed one for every thirty thousand, but each State shall have at least one Representative, and until such

\footnotetext{
${ }^{1}$ Entonces miembro de la Cámara de Representantes por el Estado de Virginia.

${ }^{2}$ Bernard Schwartz, The Great Rights of Mankind, Rowman \& Littlefield Books Inc., Maryland, 2002, pp.253-256.

${ }^{3}$ Bernard Schwartz, The Great Rights of Mankind, op. cit., pp.171.
} 
enumeration shall be made;' and that in place thereof be inserted these words, to wit: 'After the first actual enumeration, there shall be one Representative for every thirty thousand, until the number amounts to ------, after which the proportion shall never be less than ----, nor more than ------, but each State shall, after the first enumeration, have at least two representatives; and prior thereto.'”

Esta enmienda reforma el art. I, sec.2, cláusula 3, de la Constitución sobre la cual la modificación original de Madison afectaría de la siguiente manera:

\begin{abstract}
"Representatives and direct Taxes shall be apportioned among the several States which may be included within this Union, according to their respective Numbers, which shall be determined by adding to the whole Number of free Persons, including those bound to Service for a Term of Years, and excluding Indians not taxed, three fifths of all other Persons. The actual Enumeration shall be made within three Years after the first Meeting of the Congress of the United States, and within every subsequent Term of ten Years, in such Manner as they shall by Law direct. "The Number of Representatives shall not exceed one for every thirty Thousand, but each State shall have at Least one Representative; and until such enumeration shall be made, ['After the first actual enumeration, there shall be one Representative for every thirty thousand, until the number amounts to ------, after which the proportion shall never be less than ----, nor more than ------, but each State shall, after the first enumeration, have at least two representatives; and prior thereto's the State of New Hampshire shall be entitled to chuse three, Massachusetts eight, Rhode-Island and Providence Plantations one, Connecticut five, New-York six, New Jersey four, Pennsylvania eight, Delaware one, Maryland six, Virginia ten, North Carolina five, South Carolina five, and Georgia three." 6
\end{abstract}

Los cambios que Madison proponía implicaban:

a) Cambiar la ratio de representación de un representante por cada 30.000 personas hasta llegar a un número, que dejaba en blanco para que el Congreso lo determinara.

b) Una vez llegado a ese tope la proporción deberia ser regulada por el Congreso. Es decir, que las reglas de proporción entre representantes y representados volverian a cambiar una vez que se alcanzara ese máximo. A partir de ahí, sería el Congreso el encargado de redactar una nueva norma para regular el prorrateo.

Para no dejarle tanto margen de maniobra al legislativo con una materia tan delicada, Madison pensaba que la enmienda constitucional debería contener unos márgenes para dicha regulación "la proporción no deberá ser nunca menos de ----, o más de ------," 7 números que también deja por determinar al Congreso, de un representante por cada 30.000 personas, después del censo. Pero sólo

\footnotetext{
${ }^{4}$ Annals of Congress, House of Representatives, 1st Congress, 1st Session, pp.451-452.

${ }^{5}$ Tachado del texto que quería sustituir Madison por la introducción entre [] de la modificación del texto original de Madison de 1789.

${ }^{6}$ US Const. Art. I, §.2, c1.3, con la modificación sugerida por Madison.

${ }^{7}$ Annals of Congress, House of Representatives, 1st Congress, 1st Session, pp.451-452.
} 
hasta que la Cámara alcanzara un número total de diputados; número que dejaba por determinar al Congreso. ${ }^{8}$

c) Añadir un número mínimo no de uno, sino de dos diputados por cada Estado, al igual que en el Senado. Dando un pie de igualdad a los estados, independientemente de su población.

\title{
2.- Reformas en el Comité de la Cámara de Representantes
}

El Comité Selecto de la Cámara de Representantes (House Select Committee) ${ }^{9}$ presentó un informe de enmiendas al texto original de Madison el 28 de julio de $1789,{ }^{10}$ en lo que afecta a las reformas del art.I, sec. 2, cl.3 de la Constitución. El texto quedaba de la siguiente forma:

\begin{abstract}
"Art. 1, sec. 2, Par. 3 - Strike out between the words, "direct" and "and until such," and instead thereof insert, "After the first enumeration there shall be one representative for every thirty thousand until the number shall amount to one hundred; after which the proportion shall be so regulated by Congress that the number of representatives shall never be less than one hundred, nor more than one hundred and seventy-five, but each State shall always have at least one Representative." 11
\end{abstract}

Sólo dos de las tres propuestas originales de Madison quedaron plasmadas en el texto del Comité. Estas reformas a su vez pueden dividirse en cuatro partes:

1) La primera de ellas propone que se elimine del texto constitucional original la parte que constituía un Congreso provisional "hasta la realización del primer censo" 12 como ilustra la figura 1. Debido a que la función inicial del reparto del artículo I de la Constitución, ya se habría completado, al haber prorrateado los asientos del $1^{\text {er }}$ Congreso de los Estados Unidos de América. Sin embargo, como bien dice el texto constitucional, se diseñó con una intención temporal hasta la realización del primer censo decenal, que tendría lugar en 1790, tras el cual, el número de asientos correspondiente a cada estado debería reajustarse. Atendiendo a los criterios constitucionales de proporcionalidad según su población, y sin olvidar que ningún estado puede quedar sin representación en la Cámara baja.

\footnotetext{
${ }^{8}$ Bernard Schwartz, The Great Rights of Mankind, op. cit., p.257.

${ }^{9}$ Comité creado para tratar el asunto de las enmiendas, a propuesta de Madison, por tratarse del primer Congreso. Lo que justifica la inexistencia de comités permanentes y la necesidad de crear este comité ad hoc.

${ }_{11}^{10}$ Annals of Congress, op., cit., p.699.

${ }_{11}$ Annals of Congress, op., cit., p.747.

${ }^{12}$ US Const. Art. I, §.2, cl.3
} 


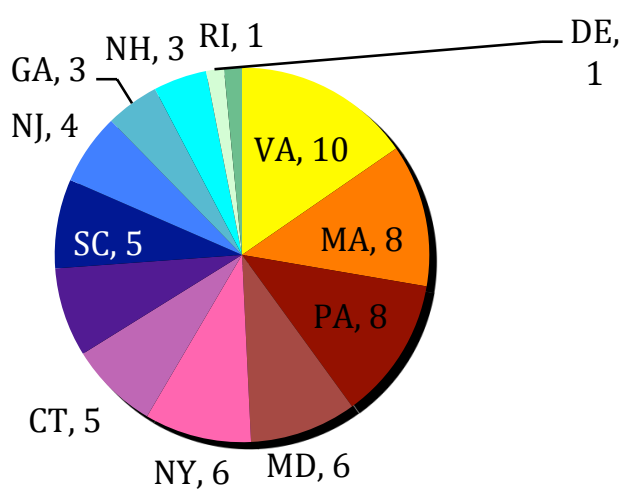

Figura 1 Distribución de Escaños en el 1er Congreso EEUU13

Técnicamente, no puede eliminarse nada del texto constitucional original. Además, lo que se aprueba hacer con la Carta de Derechos Fundamentales es añadir una lista de Derechos a la Constitución. En ningún momento retocar el texto original, puesto que significaría reabrir el debate recientemente cerrado por la Convención de Philadelphia apenas hace dos años. Lo único que pueden hacer las enmiendas es dejar sin efecto una parte del texto fundacional que, ciertamente, es el propósito de esta reforma en particular.

2) La segunda parte completa la propuesta (a) de Madison estableciendo un tope inicial de 100 diputados, para la proporción de 1 representante por cada 30.000 habitantes. ${ }^{14}$

3) La tercera parte también está inspirada por la idea original (b) de Madison de delimitar los márgenes sobre los que podrian operar las futuras leyes de prorrateo, cuya competencia correspondería al Congreso una vez superado el umbral de los 100 diputados. Para ello el Comité completa el texto original de Madison con los límites marcados entre 100 y 175 diputados. ${ }^{15}$

4) En la cuarta y última parte, el Comité rechaza la propuesta madisoniana de un mínimo de dos representantes por estado, volviendo al original constitucional de uno. Puesto que el texto del Comité vuelve a contener en su redacción que "cada estado deberá tener siempre al menos un representante." 16

El Comité decidió que el total de la Cámara subiría innecesariamente con dos representantes por Estado puesto que para mantener una representación equitativa de todos los Estados ya estaba el Senado. Si se mantenía la propuesta inicial de Madison, no sólo se aumentaban los números de la Cámara de Representantes de manera innecesaria, debido a que la función de ésta era representar a la población, por lo tanto con un representante por estado para que no hubiera ningún estado que se quedara sin representación sería suficiente. De lo contrario, el Senado carecería de sentido y el Congreso podría verse reducido a un sistema unicameral en el cual únicamente tuviéramos una Cámara de Representantes con las dos funciones.

\footnotetext{
${ }^{13}$ Gráfico de elaboración propia a partir de U.S. Const. Art.I,§.2, cl.3.

${ }^{14}$ Annals of Congress, op., cit., p.747.

${ }^{15}$ Ibid.

${ }^{16}$ Bernard Schwartz, The Great Rights of Mankind, op. cit., p.257.
} 
El Comité terminó su trabajo de revisión en una semana. El 28 de julio, presentó su informe, con las modificaciones realizadas al texto original de Madison. ${ }^{17}$

\section{3.- Votación en el pleno de la Cámara de Representantes}

Una vez terminado el trabajo del comité, Madison tuvo que convencer al resto de miembros de la Cámara, ${ }^{18}$ para someterlo a una votación en el pleno de la misma.

Esto dio lugar a un debate en el pleno de la Cámara de Representantes que duró del 18 hasta el 24 de agosto. ${ }^{19}$ Durante este debate se discutió en sesión plenaria el proyecto presentado por el Comité, al cual el conjunto de la Cámara realizó a su vez pequeñas modificaciones.

El texto en materia de prorrateo de escaños permaneció sin modificar, pero la propuesta total de enmiendas ascendió de las nueve presentadas inicialmente a diecisiete. ${ }^{20}$

\section{III.- TRAMITACIÓN LEGISLATIVA EN LA SEGUNDA CÁMARA DEL CONGRESO (SENADO)}

Una vez aprobado por el pleno de la primera cámara del Congreso, estaba listo para pasar a ser debatido por la segunda Cámara del Congreso, en este caso por el Senado. En aquel entonces a diferencia de lo que ocurría con las deliberaciones de la Cámara de Representantes, las sesiones del Senado se mantenian a puerta cerrada, ${ }^{21}$ por lo que no existe registro de las sesiones deliberativas de las enmiendas a la Constitución en su paso por la Cámara Alta.

De lo único que sí quedó constancia es del texto aprobado el día 9 de septiembre de $1789,{ }^{22}$ que reducía la propuesta de la Cámara de 17 a sólo 12 artículos. ${ }^{23}$ Quedando el texto del Senado, en referencia al prorrateo, con la siguiente forma:

"Article the first. After the first enumeration, required by the first article of the Constitution, there shall be one Representative for every thirty thousand, until that number shall amount to one hundred; to which number one Representative shall be added for every subsequent increase of forty thousand, until the Representatives shall amount to two hundred, to which number one Representative shall be added for every subsequent increase of sixty thousand persons." 24

\footnotetext{
${ }^{17}$ Annals of Congress, op., cit., p.699.

${ }_{18}^{18}$ Bernard Schwartz, The Great Rights of Mankind, op. cit., p.172.

${ }^{19}$ Annals of Congress, op., cit., pp.786-809.

${ }^{20}$ Bernard Schwartz, The Great Rights of Mankind, op. cit., pp.253-263.

${ }^{21}$ Bill Of Rights: Primary Documents Of American History, (Virtual Programs \& Services, Library of Congress), Library of Congress, http:/www.loc.gov/rr/program/bib/ourdocs/billofrights.html (último acceso: 4 de septiembre de 2014).

${ }^{22}$ Bernard Schwartz, The Great Rights of Mankind, op. cit., pp.264.

23 "Appendix: proposed amendments to the Constitution", Journal of the Senate,Vol. I, pp.96-97.

${ }^{24}$ Ibid.
} 
Tras la reforma acometida en el Senado el articulo sobre el prorrateo de la cámara experimenta las siguientes modificaciones:

i) Pasa de ser el segundo artículo en el texto de la Cámara de Representantes, a ser el primero en el texto del Senado.

ii) Cambia el límite máximo en los parámetros de representación desde 175 hasta 200 diputados.

iii) También cambia los parámetros de representación. Después explica que para estos limites lo que ha de hacerse es añadir un representante más por cada 40.000 habitantes hasta completar el nuevo número máximo (200 diputados).

iv) Una vez alcanzados los 200 diputados, se añade un representante por cada 60.000 habitantes, sin establecer más límites. Esta es una novedad del texto del Senado puesto que la cámara baja no especificaba como debía crecer la representación a partir de 175 diputados.

\section{COMISIÓN MIXTA}

El 23 de septiembre, el Senado recomendó a la Cámara de Representantes que aceptara las modificaciones que se habian hecho al texto. ${ }^{25} \mathrm{Sin}$ embargo, la Cámara de Representantes no estaba dispuesta a someterse a la imposición del Senado, por lo que hubo que convocar una comisión mixta, integrada por miembros de ambas cámaras, para pulir las diferencias de lenguaje entre los textos aprobados por ambas la Cámara de Representantes y el Senado.

Finalmente el 25 de septiembre de 1789 se aprobó un texto consensuado por ambas cámara del Congreso, con un contenido de doce enmiendas a la Constitución.

Entre ellas se encontraba el artículo referente al prorrateo, con la misma numeración que había adquirido en su texto del Senado, y su redacción final quedó de la siguiente manera:

"ARTICLE I. After the first enumeration, required by the first article of the Constitution, there shall be one Representative for every thirty thousand, until the number shall amount to one hundred, after which the proportion shall be so regulated by Congress, that there shall be not less than one hundred Representatives, nor less than one Representative for every forty thousand persons, until the number of Representatives shall amount to two hundred; after which the proportion shall be so regulated by Congress, that there shall not be less than two hundred Representatives, nor more than one Representative for every fifty thousand persons." 26

Por lo que nos interesa de estas reformas el artículo I, en su redacción final, contenía las siguientes reglas:

Después de que se lleve a cabo el primer censo: 27

\footnotetext{
${ }^{25}$ Bernard Schwartz, The Great Rights of Mankind, op. cit., p.185.

26 "Proposed amendments not ratified by the States", US Government Printing Office, 1992. http://www.gpo.gov/fdsys/pkg/GPO-CONAN-1992/pdf/GPO-CONAN-1992-8.pdf

${ }^{27}$ Resumen a partir de Enmienda I obtenida de Bernard Schwartz, The Great Rights of Mankind, op. cit., pp.264.
} 
a) $\leq 100$ diputados: 1 representante por cada 30.000 habitantes

b) Entre 100 y 200 diputados: Regulación del Congreso, manteniendo lo anterior +1 representante por cada 40.000 habitantes.

c) > 200 diputados: Nueva regulación, manteniendo todo lo anterior +1 representante por cada 50.000 habitantes.

\section{V.- APROBACIÓN DEL TEXTO FINAL}

Sometido el nuevo texto, fruto de la comisión mixta, a votación en el pleno de la Cámara de Representantes, el día 24 de septiembre de 1789, fue aprobado con 37 votos a favor y 14 en contra. ${ }^{28}$ Al día siguiente, fue votado y aprobado en el pleno del Senado. ${ }^{29}$ Por esta razón se toma esta última fecha como referencia de la aprobación de la Carta de Derechos Fundamentales (Bill of Rights) por el Congreso.

El 2 de octubre de 1789, el Presidente George Washington dio el visto bueno al proyecto de reforma constitucional aprobado a finales de septiembre por el Congreso. ${ }^{30}$

\section{VI.- RATIFICACIÓN}

El Presidente George Washington escribió cartas a todos los gobernadores de los estados que entonces formaban parte de la Unión, para que sometieran el texto a la ratificación a través de sus asambleas legislativas, ${ }^{31}$ y se necesitaría la aprobación de $3 / 4$ de los estados.

El 15 de Diciembre de 1789 sólo diez de las doce enmiendas enviadas a los estados para su ratificación fueron devueltas al Congreso ratificadas por los Estados. Los artículos no ratificados eran los dos primeros del texto enviado por el Congreso a los Estados, lo que significa que entre ellos se encontraba el artículo referente al prorrateo y el preámbulo.

Por ello lo que hoy conocemos como Carta de Derechos Fundamentales, son los diez últimos artículos de los doce iniciales.

Al quedar excluidos los dos primeros artículos se modificó la numeración de todos los artículos corriendo todos los números dos posiciones. De este modo lo que en el texto conjunto del Congreso que se envió para ser ratificado figuraba como artículo III, según la nueva numeración pasa a ser el artículo I; y así sucesivamente, hasta conformar los 10 articulos que componen "the Bill of Rights" o Carta de Derechos Fundamentales que fue Ratificada y entró en vigor en el siglo XVIII.

\footnotetext{
${ }^{28}$ Annals of Congress, op., cit., p.948.

${ }^{29}$ Bernard Schwartz, The Great Rights of Mankind, op. cit., p.186.

${ }^{30}$ Ibid.

31 "Circular to the Governors of the States, 2 October 1789," Founders Online, National Archives (http://founders.archives.gov/documents/Washington/05-04-02-0087 [last update: 2015-09-29]). Source: The Papers of George Washington, Presidential Series, vol. 4, 8 September 1789-15 January 1790, ed. Dorothy Twohig. Charlottesville: University Press of Virginia, 1993, pp. 125-127.
} 
Dicho reparto quedaría obsoleto al no incorporarse el previsto "artículo I" a la Constitución, y al no contemplar la Constitución un reparto más allá del establecido según la figura 1, "hasta la realización del primer censo." 32

No haber ratificado el "artículo I" de la propuesta en 1789 no quiere decir que no se pueda hacer en cualquier momento. Técnicamente la enmienda al quedar aprobada por el Congreso pero no ser ratificada por los Estados pasa a un 'limbo jurídico' en el que está aprobada pero no llegará a entrar en vigor a menos que efectivamente, se termine su proceso de ratificación. Esto fue lo que le ocurrió al "artículo II" del mismo texto, que sin haber conseguido la ratificación inicial en 1789, en 1992 fue rescatado y finalmente ratificado convirtiéndose así en la enmienda XXVII de la Constitución de los EEUU. En realidad, la enmienda estaba 'clinicamente muerta', porque se establece un plazo para la ratificación de las enmiendas. En caso de no cumplirlo, los textos aprobados pero no ratificados pasan a ese 'limbo jurídico' del que pueden ser resucitados. Como aconteció en 1992 con la enmienda XXVII, aunque este caso es excepcional puesto que es la única vez que se ha reavivado una enmienda fría y se ha logrado su ratificación final.

A pesar de haberse quedado a tan solo un estado de haber sido ratificada en su momento, ${ }^{33}$ es prácticamente seguro que el artículo primero del texto original no correrá la misma suerte debido a que provocaría un cambio total en el sistema político aumentando drásticamente el número de miembros de la Cámara de Representantes, y por ende el total del Congreso.

Si algunos ciudadanos americanos ya piensan que 435 miembros es un número muy elevado de congresistas, no aprobarán un texto que eleve casi 15 veces más ese número, a pesar de que, por un lado, estarian los intereses de los distritos mejor representados ¿pero sería esto cierto?

En realidad no. Puesto que una vez en Washington al no reformar el engranaje del sistema legislativo, más representantes significa más caos, y más ineficacia para el sistema.

Puesto que no se ratificó el artículo que reformaba el modelo de reparto de escaños en el Congreso, una vez que en 1790 se realizara el primer censo el, art.I, sec. 2, cl.3 y su reparto proporcional atendiendo a los criterios expuestos en la figura 1, quedaría obsoleto. Porque como bien indica la letra del articulado, el reparto que figura en la Constitución es provisional "hasta la realización del censo." 34

Por tanto, al no contemplar la Carta de Derechos Fundamentales con un nuevo sistema de reparto de escaños, la puerta siguió quedando abierta a la libre interpretación sobre cómo debía realizarse el prorrateo de escaños después de 1790. Ello ha dado lugar a que cada 10 años, con los resultados del nuevo censo, el Congreso haya tratado de regular el asunto de manera permanente sin éxito, hasta la ley de prorrateo automática de 1929.

\footnotetext{
${ }^{32}$ US Const. Art.I, $\S .2, \mathrm{cl} .3$.

${ }^{33}$ James Evan Shaw, "The Electoral College and Unstable Congressional Apportionment", en Joint Resolution Proposing an Amendment to the Constitution to Provide for the Direct Popular Election of the President and Vice President of the United States, de "Hearings Before the Subcommittee on the Constitution, of the Committee on the Judiciary," US Senate, 96th Congress, 1st session, US Government Printing Office, Washington DC, 1979, pp.463-476. ${ }^{34}$ US Const. Art.I, $\S .2, \mathrm{cl} .3$.
} 
De haber sido ratificado el artículo sobre prorrateo, habria reformado la Constitución de tal manera que los escaños al Congreso crecería cada 50.000 habitantes, "un número tan bajo que en la actualidad la Cámara de Representantes habria crecido hasta muchos miles de miembros." 35

Con los datos del censo de 2010, el tamaño de la Cámara baja sería de 6.184 de haberse aprobado la enmienda. A lo que aún queda por añadir los 100 miembros del Senado. Lo que haria un total de 6.284 miembros totales del Congreso. Número sin duda inmanejable que no habría podido llegar a crecer tanto sin llegar a enmendarse.

Fecha de envío / Submission Date: 29-10-2015

Fecha de aceptación / Acceptance Date: 21-01-2016

${ }^{35}$ Donald A. Ritchie and Justicelearning.org, Our Constitution, Oxford University Press, New York, 2006, p.11 